\title{
Variational iteration method combined with new transform to solve fractional partial differential equations
}

\author{
Mountassir Hamdi Cherif ${ }^{* *}$ and Djelloul Ziane ${ }^{\mathrm{b}}$ \\ ${ }^{a}$ Department of Mathematics and Informatics, Electrical and Energy Engineering Graduate School (ESGEE), Oran, Algeria \\ ${ }^{\mathrm{a}, \mathrm{b}}$ Laboratory of mathematics and its applications (LAMAP), University of Oran1 Ahmed Ben Bella, Oran, 31000, Algeria \\ *Corresponding author E-mail: mountassir27@yahoo.fr
}

\section{Article Info}

Keywords: Aboodh transform, Caputo fractional derivative, Diffusion equation, Klein-Gordon equation, Variational iteration method

2010 AMS: 26A33, 44A05, 35C10

Received: 20 February 2018

Accepted: 09 April 2018

Available online: 26 June 2018

\begin{abstract}
The aim of this paper is to combined the variational iteration method with Aboodh transform method to solve linear and nonlinear fractional partial differential equations. Some illustrative examples are given as the linear and nonlinear fractional Klein-Gordon equations and the time fractional diffusion equation. The results reveal that this method is very effective, simple and can be applied to other physical differential equations with fractional order. The fractional derivative is taken in the Caputo sense.
\end{abstract}

\section{Introduction}

Fractional calculus has successfully been used to study the mathematical and physical problems arising in science and engineering. Fractional differential equations are applied to describe the dynamical systems in physics and engineering. It is one of the hot topics for finding the solutions for the fractional differential equations for scientists and engineers. Due to the importance of knowledge of the solutions of these type of equations, we find that many researchers have done and are still doing great efforts to find methods to solve this type of equations. These efforts resulted in the consolidation of this research field in many methods, among them we find the homotopy analysis method ([28], [29]), Adomian decomposition method ([7], [8]), variational iteration method (VIM) ([12], [14]) and homotopy perturbation method ([13], [15]), which have become known in a large number of researchers in this area. Recently, a new option has appeared, including the composition of some transform methods with the previously mentioned methods to facilitate and improve the resolution speed of this type of equations. For example, we only mention some of these transform methods, such as Laplace transform method [11], sumudu transform method [2] or Aboodh transform method [20]. Among wich are the Laplace homotopy analysis method [25], Adomian decomposition method coupled with Laplace transform method [27], variational iteration method coupled with Laplace transform method [4], homotopy perturbation transform method [30], homotopy analysis Sumudu transform method [31], modified fractional homotopy analysis transform method [21], Sumudu decomposition method for nonlinear equations [5], variational iteration Sumudu transform method [3], homotopy perturbation Sumudu transform method [16], Aboodh decomposition method [26], fractional Aboodh decomposition method [22], Aboodh transform homotopy perturbation method [19].

The objective of this study is to combine two powerful methods, the first method is " variational iteration method", the second is called "the Aboodh transform method", for solving linear and nonlinear fractional partial differential equations, thus, we get the modified method "fractional variational iteration Aboodh transform method" (FVIATM). Several examples are given to re-confirm the effeciency of the suggested algorithm, the fractional derivative is described in this study in the sense of Caputo.

\section{Preliminaries}

In this section, we give some basic notions about fractional calculus, Aboodh transform and Aboudh transform of fractional derivatives which are used further in this paper. 


\subsection{Fractional calculus}

We give some basic definitions and properties of the fractional calculus theory as the Riemann-Liouville fractional integrals and Caputo fractional derivative (see [10], [17]).

Definition 2.1. Let $\Omega=[a, b](-\infty<a<b<+\infty)$ be a finite interval on the real axis $\mathbb{R}$. The Riemann-Liouville fractional integral $I_{0+}^{\alpha} f$ of order $\alpha \in \mathbb{R}(\alpha>0)$ is defined by

$$
\begin{aligned}
& \left(I_{0+}^{\alpha} f\right)(\tau)=\frac{1}{\Gamma(\alpha)} \int_{0}^{\tau} \frac{f(\varsigma) d \varsigma}{(\tau-\varsigma)^{1-\alpha}}, \quad \tau>0, \alpha>0 \\
& \left(I_{0+}^{0} f\right)(\tau)=f(\tau)
\end{aligned}
$$

Here $\Gamma(\cdot)$ is the gamma function.

Theorem 2.2. Let $\alpha \geq 0$ and let $n=[\alpha]+1$. If $f(\tau) \in A C^{n}[a, b]$, then the Caputo fractional derivative $\left({ }^{c} D_{0+}^{\alpha} f\right)(\tau)$ exist almost evrywhere on $[a, b]$. If $\alpha \notin \mathbb{N},\left({ }^{c} D_{0^{+}}^{\alpha} f\right)(\tau)$ is represented by

$$
\left({ }^{c} D_{0+}^{\alpha} f\right)(\tau)=\frac{1}{\Gamma(n-\alpha)} \int_{0}^{\tau} \frac{f^{(n)}(\varsigma) d \varsigma}{(\tau-\varsigma)^{\alpha-n+1}},
$$

where $D=\frac{d}{d r}$ and $n=[\alpha]+1$.

Remark 2.3. In this paper, we consider the time-fractional derivative in the Caputo's sense. When $\alpha \in \mathbb{R}^{+}$, the time-fractional derivative is defined as

$$
\begin{aligned}
\left({ }^{c} D_{\tau}^{\alpha} u\right)(r, \tau) & =\frac{\partial^{\alpha} u(r, \tau)}{\partial \tau^{\alpha}} \\
& =\left\{\begin{array}{r}
\frac{1}{\Gamma(m-\alpha)} \int_{0}^{\tau}(\tau-\varsigma)^{m-\alpha-1} \frac{\partial^{m} u(r, \zeta) d \varsigma}{\partial \varsigma^{m}}, m-1<\alpha<m, \\
\frac{\partial^{m} u(r, \tau)}{\partial \tau^{m}}, \quad \alpha=m,
\end{array}\right.
\end{aligned}
$$

where $m \in \mathbb{N}^{*}$.

\subsection{Definitions and properties of the Aboodh transform}

The Aboodh transform was defined by K. S. Aboodh [20] in 2013. In this section, we give some basic definitions and properties of this transform (see [1], [18], [20]).

\subsubsection{Definitions}

The Aboodh transform is defined for functions of exponential order. We consider functions belonging to a class $B$, where $B$ defined by

$$
B=\left\{u(\tau):|u(\tau)|<M e^{k_{j}|\tau|}, \text { if } \tau \in(-1)^{j} \times\left[0, \infty, j=1,2 ; M, k_{1}, k_{2}>0\right\} .\right.
$$

Definition 2.4. The Aboodh integral transform of the function $u$ in $B$ is defined by the integral equation

$$
A[u(\tau)]=U(v)=\frac{1}{v} \int_{0}^{\infty} u(\tau) e^{-v \tau} d \tau ; \tau \geq o, v \in\left(k_{1}, k_{2}\right)
$$

The variable $v$ in this transform is used to factor the variable $\tau$ in the argument of the function $u$.

Proposition 2.5. The Aboodh transform of the time-fractional derivative in the Caputo's sense is defined as

$$
A\left[\left({ }^{c} D_{0+}^{\alpha} u\right)(\tau) ; v\right]=v^{\alpha} A[u(\tau)]-\sum_{k=0}^{n-1} \frac{u^{(k)}(0)}{v^{2-\alpha+k}}, n-1<\alpha \leq n, n=1,2, \ldots
$$

And the Aboodh transform of the function $u(r, \tau)$ with Caputo fractional derivative of order $\alpha$ is given by

$$
A\left[\left({ }^{c} D_{0+}^{\alpha} u\right)(r, \tau) ; v\right]=v^{\alpha} A[u(r, \tau)]-\sum_{k=0}^{n-1} \frac{u^{(k)}(r, 0)}{v^{2-\alpha+k}}, n-1<\alpha \leq n, n=1,2, \ldots
$$




\subsubsection{Somme properties of the Aboodh transform}

1. The Aboodh transform of the $n t h$ derivative of $u(\tau)$ is given by

$$
A\left[u^{(n)}(\tau)\right]=U_{n}(v)=v^{n} A[u(\tau)]-\sum_{k=0}^{n-1} \frac{u^{(k)}(0)}{v^{2-n+k}}
$$

2. Some elementary functions and their transformations

\begin{tabular}{|l|l|}
\hline$u(\tau)$ & $A[u(\tau)]$ \\
\hline 1 & $\frac{1}{v^{2}}$ \\
\hline$\tau$ & $\frac{1}{v^{3}}$ \\
\hline$\tau^{n}$ & $\frac{n !}{v^{n+2}}, n=0,1,2, \ldots$ \\
\hline$\tau^{\alpha}$ & $\frac{\Gamma(\alpha+1)}{v^{\alpha+2}}, \alpha \geq 0$. \\
\hline
\end{tabular}

\section{Analysis of fractional variational iteration Aboodh transform method (FVIATM)}

To illustrate the basic idea of this method, we consider a general nonlinear partial differential equation of fractional order

$$
{ }^{c} D_{\tau}^{\alpha} U(r, \tau)+R U(r, \tau)+N U(r, \tau)=g(r, \tau)
$$

where $m-1<\alpha \leq m, m=1,2, \ldots$ and the initial conditions

$$
\left[\frac{\partial^{m-1} U(r, \tau)}{\partial \tau^{m-1}}\right]_{\tau=0}=h_{m-1}(r)
$$

where ${ }^{c} D_{\tau}^{\alpha}=\frac{\partial^{\alpha}}{\partial \tau^{\alpha}}$ is the Caputo fractional derevative, $R$ is the linear differential operator, $N$ represents the general nonlinear differential operator, and $g(r, \tau)$ is the source term.

Applying Aboodh transform on both sides of (3.1), we obtain

$$
A\left[{ }^{c} D_{\tau}^{\alpha} U(r, \tau)\right]+A[R U(r, \tau)]+A[N U(r, \tau)]=A[g(r, \tau)]
$$

Using the differentiation property of Aboodh transform, we have

$$
A[U(r, \tau)]=\frac{1}{v^{\alpha}} \sum_{k=0}^{n-1} \frac{U^{(k)}(r, 0)}{v^{2-\alpha+k}}+\frac{1}{v^{\alpha}} A[g(r, \tau)]-\frac{1}{v^{\alpha}} A[R U(r, \tau)+N U(r, \tau)] .
$$

Operating with the inverse Aboodh transform on both sides of (3.4), we obtain

$$
U(r, \tau)=H(r, \tau)-A^{-1}\left(\frac{1}{v^{\alpha}} A[R U(r, \tau)+N U(r, \tau)]\right)
$$

where $H(r, \tau)$, represents the term arising from the source term and the prescribed initial conditions.

Applying $\frac{\partial}{\partial \tau}$ on both sides of (3.5), we have

$$
\frac{\partial U(r, \tau)}{\partial \tau}+\frac{\partial}{\partial \tau} A^{-1}\left(\frac{1}{v^{\alpha}} A[R U(r, \tau)+N U(r, \tau)]\right)-\frac{\partial H(r, \tau)}{\partial \tau}=0
$$

According to the variational iteration method ([12], [14]), we can construct a correct functional as follows

$$
U_{n+1}(r, \tau)=U_{n}(r, \tau)-\int_{0}^{\tau}\left[\frac{\partial U_{n}(r, \varsigma)}{\partial \varsigma}+\frac{\partial}{\partial \varsigma} A^{-1}\left(\frac{1}{v^{\alpha}} A\left[R U_{n}(r, \varsigma)+N U_{n}(r, \varsigma)\right]\right)-\frac{\partial H(r, \varsigma)}{\partial \varsigma}\right] d \varsigma
$$

Recall that $U(r, \tau)=\lim _{n \rightarrow \infty} U_{n}(r, \tau)$.

That may give the exact solution if a closed form one exists, or we can use the $(n+1)$ th approximation for numerical purposes. The convergence of the variational iteration method is introduit by Tatari et all. in [24]. Though the variational iteration method leads to fast convergent solutions, unnecessary calculation arises in the solution procedure. 


\section{Applications}

To illustrate the efficiency of the fractional variational iteration Aboodh transform method, we apply this method to solve some linear and nonlinear time-fractional partial differential equations with Caputo fractional derivative.

Example 4.1. Consider the following time fractional diffusion equation

$$
\begin{gathered}
{ }^{c} D_{\tau}^{\alpha} U(r, \tau)=\frac{r^{2}}{2} U_{r r}(r, \tau), \quad 0<\alpha \leq 1 \\
U(r, 0)=r^{2}
\end{gathered}
$$

and which subject to the boundary conditions $U(0, \tau)=0$ and $U(1, \tau)=f(\tau)$.

Applying Aboodh transform on both sides of (4.1) and using its differentiation property, we obtain

$$
A[U(r, \tau)]=\frac{1}{v^{2}} r^{2}+\frac{1}{v^{\alpha}} A\left[\frac{r^{2}}{2} U_{r r}(r, \tau)\right] .
$$

Taking the inverse Aboodh transform of (4.2), we have

$$
U(r, \tau)=r^{2}+A^{-1}\left(\frac{1}{v^{\alpha}} A\left[\frac{r^{2}}{2} U_{r r}(r, \tau)\right]\right)
$$

Applying $\frac{\partial}{\partial \tau}$ on both sides of (4.3), we get

$$
\frac{\partial U(r, \tau)}{\partial \tau}=\frac{\partial}{\partial \tau} A^{-1}\left(\frac{1}{v^{\alpha}} A\left[\frac{r^{2}}{2} U_{r r}(r, \tau)\right]\right) .
$$

According to the variational iteration method, we can construct a correct functional as follows

$$
U_{n+1}(r, \tau)=U_{n}(r, \tau)-\int_{0}^{\tau}\left[\frac{\partial U_{n}(r, \varsigma)}{\partial \varsigma}-\frac{\partial}{\partial \varsigma} A^{-1}\left(\frac{1}{v^{\alpha}} A\left[\frac{r^{2}}{2}\left(U_{n}\right)_{r r}(r, \tau)\right]\right)\right] d \varsigma .
$$

By using the iteration formula (4.5), the first terms are given by

$$
\begin{gathered}
U_{0}(r, \tau)=r^{2}, \\
U_{1}(r, \tau)=r^{2}+r^{2} \frac{\tau^{\alpha}}{\Gamma(\alpha+1)}, \\
U_{2}(r, \tau)=r^{2}+r^{2} \frac{\tau^{\alpha}}{\Gamma(\alpha+1)}+r^{2} \frac{\tau^{2 \alpha}}{\Gamma(2 \alpha+1)}, \\
U_{3}(r, \tau)=r^{2}+r^{2} \frac{\tau^{\alpha}}{\Gamma(\alpha+1)}+r^{2} \frac{\tau^{2 \alpha}}{\Gamma(2 \alpha+1)}+r^{2} \frac{\tau^{3 \alpha}}{\Gamma(3 \alpha+1)} \\
\vdots \\
U_{n}(r, \tau)=\sum_{k=0}^{n} \frac{r^{2} \tau^{k \alpha}}{\Gamma(k \alpha+1)} .
\end{gathered}
$$

Recall that the solution is given by

$$
U(r, \tau)=\lim _{n \rightarrow \infty} U_{n}(r, \tau)=\lim _{n \rightarrow \infty} \sum_{k=0}^{n} \frac{r^{2} \tau^{k \alpha}}{\Gamma(k \alpha+1)}=r^{2} E_{\alpha}\left(\tau^{\alpha}\right)
$$

which is the exact solution of time fractional diffusion equation (4.1) obtained by fractional variational iteration method in [9], but with less calculations. In the case $\alpha=1$, it is given by $U(r, \tau)=r^{2} e^{\tau}$.

Example 4.2. Consider the linear fractional Klein-Gordon equation

$$
{ }^{c} D_{\tau}^{\alpha} U(r, \tau)=U_{r r}(r, \tau)-U(r, \tau), \quad 1<\alpha \leq 2,
$$

with the initial conditions

$$
U(r, 0)=0, U_{\tau}(r, 0)=r .
$$

By applying the Aboodh transform on both sides of (4.8), we get

$$
A[U(r, \tau)]=\frac{1}{v^{3}} r+\frac{1}{v^{\alpha}} A\left[U_{r r}(r, \tau)-U(r, \tau)\right]
$$




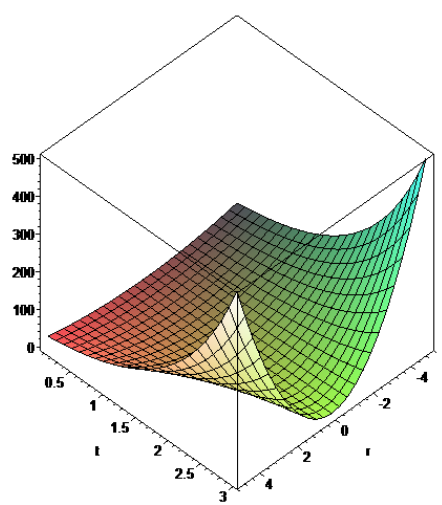

(a)

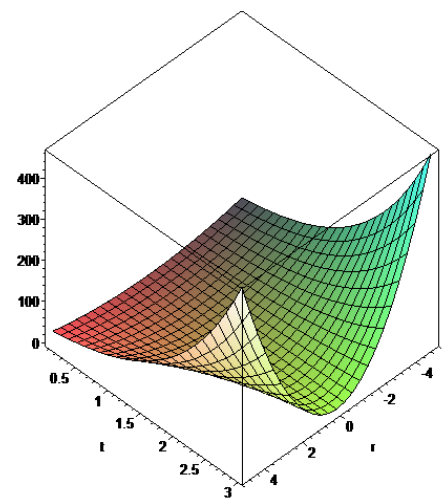

(b)

Figure 4.1: (a) The exact solution, (b) The approximate solution when $\alpha=1$ of (4.1).

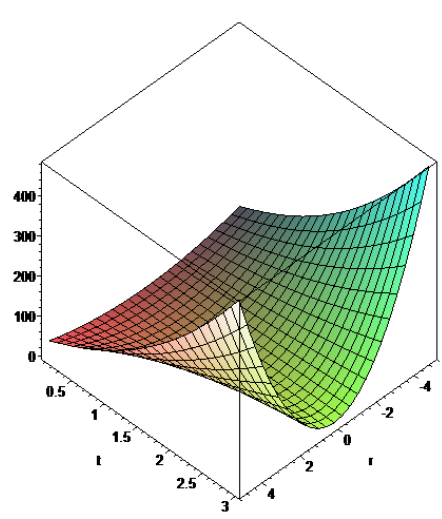

(c)

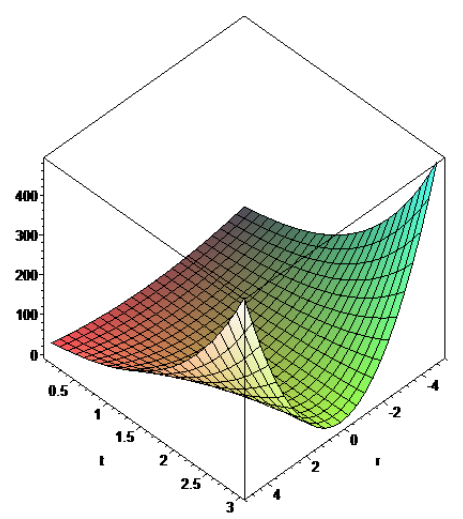

(d)

Figure 4.2: (c) and (d) The approximate solutions of (4.1) when $\alpha=0.5$ and $\alpha=0.9$ respectively.

Taking the inverse Aboodh transform of (4.10), we have

$$
U(r, \tau)=r \tau+A^{-1}\left(\frac{1}{v^{\alpha}} A\left[U_{r r}(r, \tau)-U(r, \tau)\right]\right) .
$$

Applying $\frac{\partial}{\partial \tau}$ on both sides of (4.11), we get

$$
\frac{\partial U(r, \tau)}{\partial \tau}=r+\frac{\partial}{\partial \tau} A^{-1}\left(\frac{1}{v^{\alpha}} A\left[U_{r r}(r, \tau)-U(r, \tau)\right]\right) .
$$

According to the variational iteration method, we can construct a correct functional as follows

$$
U_{n+1}(r, \tau)=U_{n}(r, \tau)-\int_{0}^{\tau}\left[\frac{\partial U_{n}(r, \varsigma)}{\partial \varsigma}-r-\frac{\partial}{\partial \varsigma} A^{-1}\left(\frac{1}{v^{\alpha}} A\left[U_{n r r}(r, \tau)-U_{n}(r, \tau)\right]\right)\right] d \varsigma .
$$

Consequently, the first terms are obtained by

$$
\begin{gathered}
U_{0}(r, \tau)=r \tau, \\
U_{1}(r, \tau)=r \tau-r \frac{\tau^{\alpha+1}}{\Gamma(\alpha+2)}, \\
U_{2}(r, \tau)=r \tau-r \frac{\tau^{\alpha+1}}{\Gamma(\alpha+2)}+r \frac{\tau^{2 \alpha+1}}{\Gamma(2 \alpha+2)}, \\
U_{3}(r, \tau)=r \tau-r \frac{\tau^{\alpha+1}}{\Gamma(\alpha+2)}+r \frac{\tau^{\alpha \alpha+1}}{\Gamma(2 \alpha+2)}-r \frac{\tau^{3 \alpha+1}}{\Gamma(3 \alpha+2)}, \\
\vdots \\
U_{n}(r, \tau)=r \sum_{k=0}^{n}(-1)^{k} \frac{\tau^{k \alpha+1}}{\Gamma(k \alpha+2)}
\end{gathered}
$$

The approximate solution in a series form of (4.8)-(4.9) when $\alpha \longrightarrow 2$, is given by

$$
U(r, \tau)=\lim _{n \rightarrow \infty} U_{n}(r, \tau)=r \sin \tau,
$$

which is the exact solution of linear Klein-Gordon equation presented in [23]. 


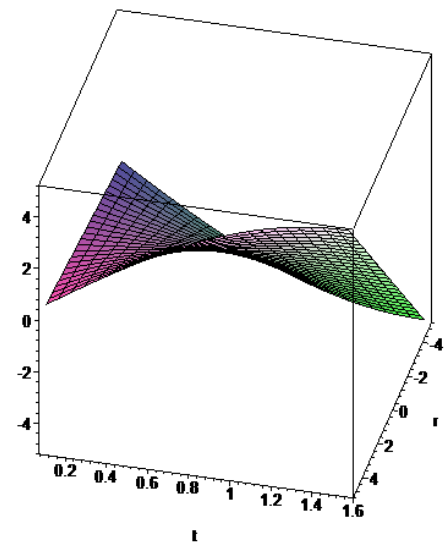

(a')

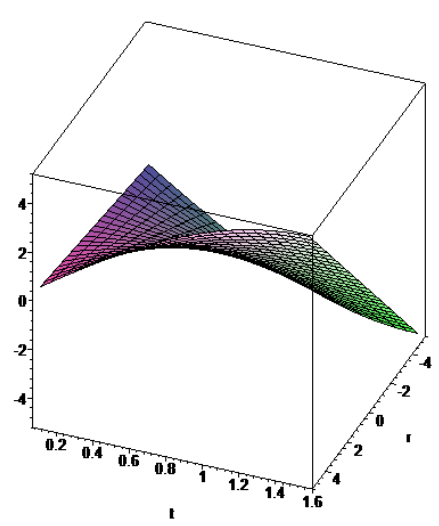

(b')

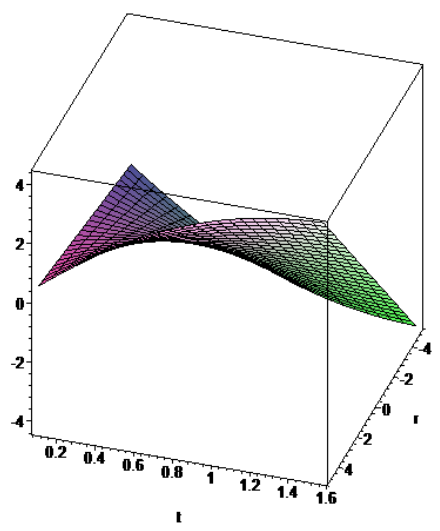

(c')

Figure 4.3: (a') The exact solution and (b') The approximate solution in the case $\alpha=2$, (c') The approximate solution when $\alpha=1.5$ of (4.8)-(4.9).

Example 4.3. We consider the nonlinear fractional Klein-Gordon equation of the form

$$
\begin{gathered}
{ }^{c} D_{\tau}^{\alpha} U(r, \tau)=U_{r r}(r, \tau)-U^{2}(r, \tau)+r^{2} \tau^{2}, \quad 1<\alpha \leq 2, \\
\\
U(r, 0)=0, U_{\tau}(r, 0)=r .
\end{gathered}
$$

Applying Aboodh transform on both sides of (4.16), we have

$$
A[U(r, \tau)]=\frac{1}{v^{3}} r+2 r^{2} \frac{1}{v^{\alpha+4}}+\frac{1}{v^{\alpha}} A\left[U_{r r}(r, \tau)-U^{2}(r, \tau)\right] .
$$

By inverse Aboodh transform and derivative, we get

$$
\frac{\partial U(r, \tau)}{\partial \tau}=r+2(\alpha+2) r^{2} \frac{\tau^{\alpha+1}}{\Gamma(\alpha+3)}+\frac{\partial}{\partial \tau} A^{-1}\left(\frac{1}{v^{\alpha}} A\left[U_{r r}(r, \tau)-U^{2}(r, \tau)\right]\right) .
$$

Now, applying the variational iteration method, we obtain

$$
U_{n+1}(r, \tau)=U_{n}(r, \tau)-\int_{0}^{\tau}\left[\frac{\partial U_{n}(r, \varsigma)}{\partial \varsigma}-r-2(\alpha+2) r^{2} \frac{\tau^{\alpha+1}}{\Gamma(\alpha+3)}-\frac{\partial}{\partial \varsigma} A^{-1}\left(\frac{1}{v^{\alpha}} A\left[U_{n r r}(r, \tau)-U_{n}^{2}(r, \tau)\right]\right)\right] d \varsigma
$$

The first terms of approximate solution are obtained successively

$$
\begin{gathered}
U_{0}(r, \tau)=r \tau+\frac{2 r^{2}}{\Gamma(\alpha+3)} \tau^{\alpha+2} \\
U_{1}(r, \tau)=r \tau+\frac{4}{\Gamma(\alpha+3) \Gamma(2 \alpha+3)} \tau^{2 \alpha+2} \\
-\frac{4 r^{3} \Gamma(\alpha+4)}{\Gamma(\alpha+3) \Gamma(2 \alpha+4)} \tau^{2 \alpha+3}-\frac{4 r^{4} \Gamma(2 \alpha+5)}{\Gamma^{2}(\alpha+3) \Gamma(3 \alpha+5)} \tau^{3 \alpha+4}
\end{gathered}
$$

and so on. Therefore the solution of (4.16) in series form when $\alpha=2$, is given by

$$
U(r, \tau)=\lim _{n \rightarrow \infty} U_{n}(r, \tau)=r \tau
$$

Example 4.4. We consider the following nonlinear time-fractional partial differential equation

$$
{ }^{c} D_{\tau}^{\alpha} U-\frac{3}{8}\left[\left(U_{r r}\right)^{2}\right]_{r}=\frac{3}{2} \tau, \quad 2<\alpha \leq 3,
$$

with the initial conditions

$$
U(r, 0)=\frac{1}{2} r^{2}, U_{\tau}(r, 0)=\frac{1}{3} r^{3}, U_{\tau \tau}(r, 0)=0 .
$$

According to the formula (3.7), we can construct the following iteration formula

$$
U_{n+1}(r, \tau)=-\frac{1}{2} \tau^{2}+\frac{1}{3} r^{3} \tau+\frac{3}{2} \frac{\tau^{\alpha+1}}{\Gamma(\alpha+2)}-A^{-1}\left(\frac{1}{v^{\alpha}} A\left[-\frac{3}{8}\left[\left(U_{n r r}\right)^{2}\right]_{r}\right]\right)
$$


Using the iteration formula (4.24), we obtain

$$
\begin{gathered}
U_{0}(r, \tau)=-\frac{1}{2} r^{2}+\frac{1}{3} r^{3} \tau, \\
U_{1}(r, \tau)=-\frac{1}{2} r^{2}+\frac{1}{3} r^{3} \tau+6 r \frac{\tau^{\alpha+2}}{\Gamma(\alpha+3)}, \\
U_{2}(r, \tau)=-\frac{1}{2} r^{2}+\frac{1}{3} r^{3} \tau+6 r \frac{\tau^{\alpha+2}}{\Gamma(\alpha+3)}, \\
U_{3}(r, \tau)=-\frac{1}{2} r^{2}+\frac{1}{3} r^{3} \tau+6 r \frac{\tau^{\alpha+2}}{\Gamma(\alpha+3)},
\end{gathered}
$$

The approximate solution in a series form, is given by

$$
U(r, \tau)=-\frac{1}{2} r^{2}+\frac{1}{3} r^{3} \tau+6 r \frac{\tau^{\alpha+2}}{\Gamma(\alpha+3)} .
$$

As $\alpha \longrightarrow 3$, we get

$$
U(r, \tau)=-\frac{1}{2} r^{2}+\frac{1}{3} r^{3} \tau+\frac{1}{20} r \tau^{5}
$$

which is an exact solution of the nonlinear partial differential equation of order three (4.22)-(4.23) obtained by the modified homotopy analysis method in [6].

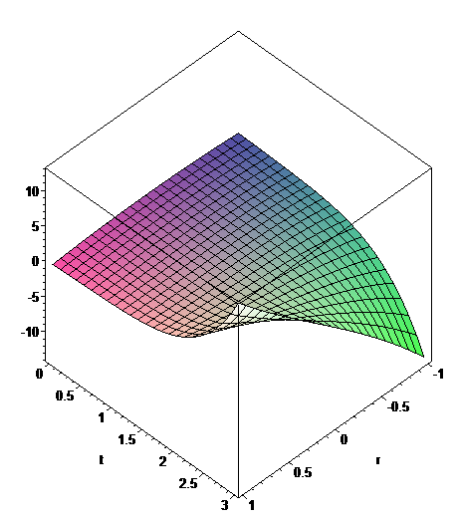

(a")

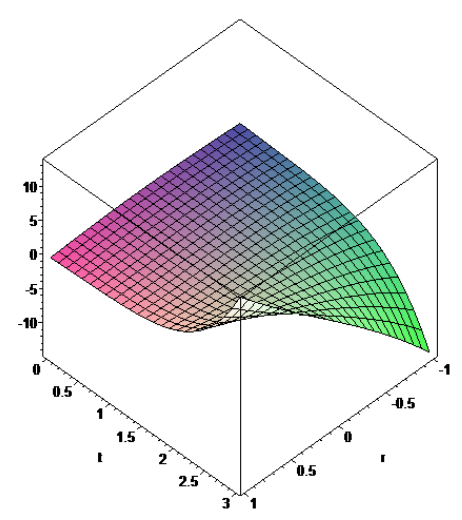

(b")

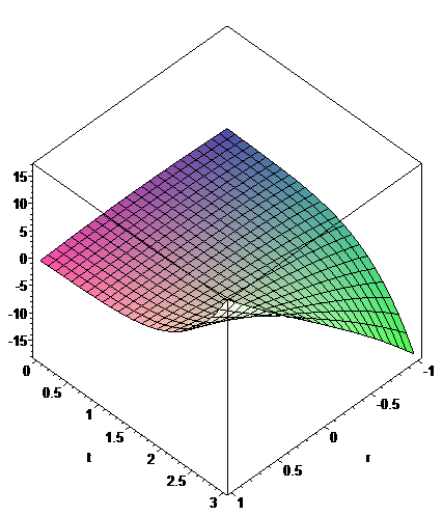

(c")

Figure 4.4: (a") The exact solution, (b") and (c") The approximate solutions in the case $\alpha=2.9$ and $\alpha=1.5$ respectively of (4.22)-(4.23).

\section{Conclusion}

In this work, a variational iteration method (VIM) and new transform method called "Aboodh transform" are successfully combined to form a powerful analytical method for solving fractional partial differential equations. The new analytical method gives a series solution which converges rapidly to the exact solution. The simplicity and high precision of the new analytical method are clearly illustrated, for example, by the resolution of some equations such as the time fractional diffusion equation, the linear and nonlinear fractional Klein-Gordon equation of order 2 and an example of nonlinear time fractional partial differential equation of order three.

\section{References}

[1] A. K. Hassan Sedeeg and M. M. Abdelrahim Mahgob, Comparison of New Integral Transform Aboodh Transform and Adomain Decomposition Method, Int. J. Math. And its Appl. 4 (2)-B (2016), 127-135.

[2] A. Kiliçman and H. Eltayeb, On a New Integral Transform and Differential Equations, Math. Problems in Eng. A. ID 463579, (2010), 13 pp.

[3] A. S. Abedl-Rady, S. Z. Rida, A. A. M. Arafa and H. R. Abedl-Rahim, Variational Iteration Sumudu Transform Method for Solving Fractional Nonlinear Gas Dynamics Equation, Int. J. Res. Stu. Sci. Eng. Tech. 1 (2014), 82-90.

[4] A. S. Arife and A. Yildirim, New Modified Variational Iteration Transform Method (MVITM) for solving eighth-order Boundary value problems in one Step, W. Appl. Sci. J. 13 (2011), $2186-2190$.

[5] D. Kumar, J. Singh and S. Rathore, Sumudu Decomposition Method for Nonlinear Equations, Int. Math. For. 7 (2012), $515-521$.

[6] D. Ziane and M. Hamdi Cherif, Modified homotopy analysis method for nonlinear fractional partial differential equations, Int. J. Anal. Appl. 14 (1), (2017), 77-87.

[7] G. Adomian, Nonlinear Stochastic Systems Theory and Applications to Physics, Kluwer Academic Publishers, Netherlands, 1989.

[8] G. Adomian, Solution of physical problems by decomposition, Comput. Math. Appl. 27 (1994), 145-154.

[9] G. Wu and E. W. M. Lee, Fractional variational iteration method and its application, Phys. Letters A. 374 (2010), $2506-2509$.

[10] I. Podlubny, Fractional Differential Equations, Academic Press, San Diego, CA, 1999, 1-365.

[11] I. Podlubny, The Laplace Transform Method for Linear Differential Equations of the Fractional Order, Slovak Acad. Sci. Inst. Exp. Phys, 1997.

[12] J. H. He, A new approach to nonlinear partial differential equations, Comm. Nonlinear. Sci. Numer. Simul. 2 (1997), 203-205.

[13] J. H. He, A new perturbation technique which is also valid for large parameters, J. Sound Vib. 229 (2000), 1257-1263.

[14] J. H. He, A variational iteration approach to nonlinear problems and its applications, Mech. Appl. 20 (1), (1998), 30-31.

[15] J. H. He, Homotopy perturbation technique, Comput. Meth. Appl. Mech. Eng. 178 (1999), 257-262. 
[16] J. Singh, D. Kumar and Sushila,Homotopy Perturbation Sumudu Transform Method for Nonlinear Equations, Adv. Theor. Appl. Mech. 4 (2011), $165-175$.

[17] K. Diethelm, The Analysis Fractional Differential Equations, Springer-Verlag Berlin Heidelberg, 2010, 1-262.

[18] K. S. Aboodh, A. Idris and R. I. Nuruddeen, On the Aboodh Transform Connections with Some Famous Integral Transforms, Int. J. Engin. Info. Syst. 1(9), (2017), 143-151.

[19] K. S. Aboodh, Solving Fourth Order Parabolic PDE with Variable Coefficients Using Aboodh Transform Homotopy Perturbation Method, Pure. Appl. Math. J. 4(5), (2015), 219-224.

[20] K. S. Aboodh, The New Integral Transform ”AboodhTransform, Glob. J. Pure. Appl. Math. 9 (1), (2013), 35-43.

[21] K. Wang and S. Liu, Application of new iterative transform method and modified fractional homotopy analysis transform method for fractional Fornberg-Whitham equation, J. Nonlinear Sci. Appl. 9 (2016), 2419-2433.

[22] M. Hamdi Cherif and D. Ziane, A New Numerical Technique for Solving Systems of Nonlinear Fractional Partial Differential Equations, Int. J. Anal. Appl. 15(2), (2017), 188-197.

[23] M. Khalid, M. Sultana, F. Zaidi and A. Uroosa,Solving linear and nonlinear Klein-Gordan equations by new perturbation iteration transform method, J. App. Eng. Math. 6(1), (2016), 115-125.

[24] M. Tatari and M. Dehghan, On the convergence of He's variational iteration method, J. Comp. Appl. Math. 207(1), (2007), 121-128.

[25] M. Zurigat, Solving Fractional Oscillators Using Laplace Homotopy Analysis Method, Annals of the University of Craiova, Math. Comp. Sci. Series. 38 (2011), 1-11.

[26] R. I. Nuruddeen and A. M Nass, Aboodh Decomposition Method and its Application in Solving Linear and Nonlinear Heat Equations, Europ. J. Advances in Engin. Techn. 3(7), (2016), 34-37.

[27] S. A. Khuri, A Laplace decomposition algorithm applied to a class of nonlinear differential equations, J. Math. Annl. Appl. 4 (2001), 141-155.

[28] S. J. Liao, On the homotopy analysis method for nonlinear problems, Appl. Math. Comput. 147 (2004), $499-513$.

[29] S. J. Liao, The proposed homotopy analysis technique for the solution of nonlinear problems, Ph.D. Thesis, Shanghai Jiao Tong University, 1992.

[30] S. Kumara, A. Yildirim, Y. Khan and L. Weid, A fractional model of the diffusion equation and its analytical solution using Laplace transform, Scientia Iranica B. 19 (2012), 1117-1123.

[31] S. Rathore, D. Kumar, J. Singh and S. Gupta, Homotopy Analysis Sumudu Transform Method for Nonlinear Equations, Int. J. Industrial Math. 4 (2012), 301-314. 